\title{
TRABAJO SOCIAL ESCOLAR DESDE LA PERSPECTIVA DE ALUMNAS Y ALUMNOS EN ALEMANIA. UN EJEMPLO DE BADEN-WÜRTTEMBERG.
}

Resumen. El Trabajo Social Escolar (Schulsozialarbeit) en Alemania se transformó desde hace aproximadamente 15 años en una de las ofertas más importante correspondientes a la Asistencia Social Juvenil (Jugenhilfe) y adquirió un perfil profesional importante. El presente aporte reconstruye a partir de una investigación cualitativa la perspectiva de los jóvenes y sus modelos subjetivos de adquisiciòn de competencias que son estructuradas a través de la organización institucional escolar. El valor de la operatividad del Trabajo Social Escolar se fundamenta en primer lugar en la relevancia de los profesionales entendidos como los portadores del sentido de la oferta de ayuda, en segundo lugar en la experiencias y relaciones realizadas en el proceso pedagógico cotidiano, en tercer lugar en la confianza de dichas referencias y en cuarto lugar a través de la experiencia se comprende que los temas correspondientes a su propia vida, adquieren significado. La interconección planteada entre la dimensión del uso y la dimensión de la empleabilidad (operatividad) confirma en quinto lugar la relevancia que posee la modulación de las relaciones pedagógicas entre las distintas generaciones como elemento estructural del Trabajo Social Escolar.

Palabras claves: Trabajo Social Escolar; escuela; investigación cualitativa, investigación de los beneficiarios; relaciones intergeneracionales.

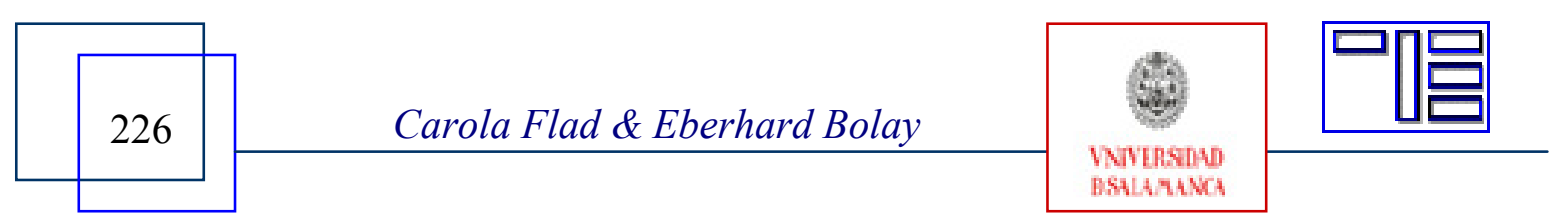




\section{SOCIAL SCHOOL WORK FROM THE STUDENT'S PERSPECTIVE IN GERMANY. AN EXAMPLE OF BADEN -WÜRTTEMBERG.}

Abstract. Over the past 15 years school social work in Germany has become one of the most important tools of youth support and has developed an acknowledged professional profile. The view of young people and their subjective patterns of acquisition as they are constructed by the institutional arrangements of schools have been reconstructed in this article by using a qualitative research approach. The value of school social work becomes obvious first of all through the professionals as relevant persons in this field of support, secondly by the social experiences in the daily pedagogical process, as well as by the reliability of these relationships and also by the fact that their daily concerns are taken seriously. The analytical crossover between the dimension of use and the dimension of usefulness (use value) hints furthermore towards the relevance of a pedagogical arrangement of relations between the generations as a structural element of school social work.

Key words: School social work, school, qualitative research, subject focussed research, generational relations.

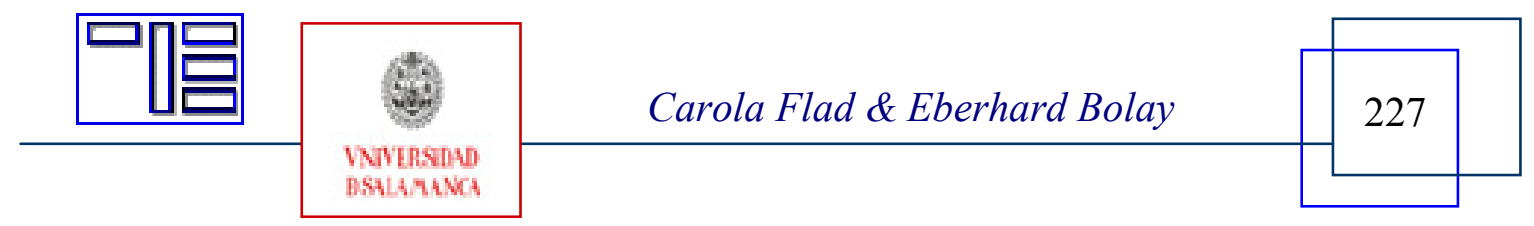




\section{TRAVAIL SOCIAL SCOLAIRE DE LA PRESPECTIVE DES ÉLÈVES EN ALLEMAGNE, UN EXEMPLE DE BADEN-WÜRTTEMBERG.}

Sommaire. Durant ces 15 dernières années, le travail social dans le cadre scolaire en Allemagne est devenu un des outils les plus important du soutien à la jeunesse et, a developpé un profil professionel reconnu.

La vision des jeunes et les manières subjectives d'aquérir cette vision, étant donné qu'elles découlent de l'arrangement institutionel des écoles, ont été rassemblées dans cette article grâce à une démarche de recherche qualitative.

La valeur du travail social dans le cadre scolaire devient évidente, tout d'abord à travers les professionels en tant que personnes appropriés pour genre de soutien aporté, deuxièmement, à travers l'expérience sociale du procesus pédagogique journalier, également grâce à la fiabilité des ces relations et, enfin, du fait que leurs soucis quotidiens soient pris au serieux. La croisée analytique entre la dimension d'utilisation et la dimension d'utilité indique d'autant plus l'importance de l'arrangement pédagogique des relations inter-générationelles, en tant qu'élément structurel du travail social en milieu scolaire.

Mots-clés: travail social en milieu scolaire, école, recherche qualitative, recherche centrée sur le sujet, relations générationelles.

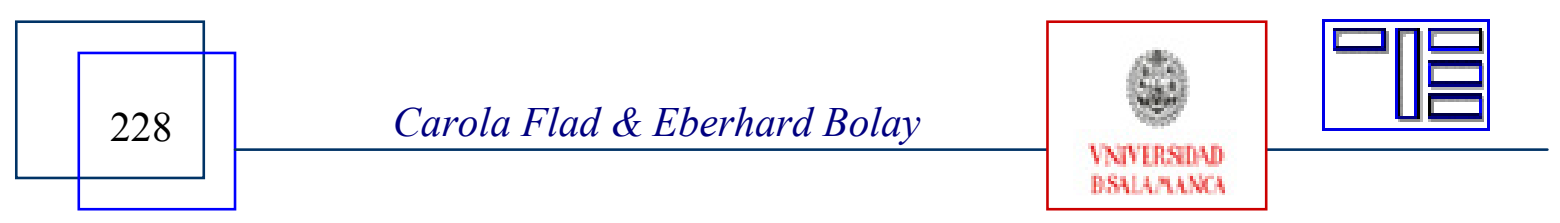


TRABAJO SOCIAL ESCOLAR DESDE LA PERSPECTIVA DE ALUMNAS Y ALUMNOS EN ALEMANIA. UN EJEMPLO DE BADEN-WÜRTTEMBERG. ${ }^{1}$

\author{
Carola Flad, Eberhard Bolay \\ carola.flad@uni-tuebingen.de; eberhard.bolay@uni-tuebingen.de \\ Universidad de Tübingen (Alemania).
}

\title{
1.- INTRODUCCIÓN.
}

Sin duda que el trabajo social en las escuelas como oferta de apoyo a los jóvenes está adquiriendo cada vez más perfil y coherencia. Esto se puede comprobar en los debates científicos al respecto (vid. Olk/Bathke/Hartnuß 2000; Maykus 2001; Bettmer et al. 2002; Bolay 2004a), al igual que en los resultados de análisis empíricos prácticos (vid. Bolay 2004b). Siendo las alumnas y los alumnos los destinatarios directos del trabajo social escolar, ellos hasta el momento sólo han tenido en forma limitada la posibilidad de articularse. En este trabajo queremos, pues, poner énfasis especialmente en la perspectiva de los jóvenes y su modelo subjetivo de apropiación de las medidas, es decir "de la reconstrucción de la perspectiva de los destinatarios" hacia esa oferta de apoyo juvenil en escuelas. Tal perspectiva requiere del "análisis de los entornos (Umwelten) sociales e institucionales que los rodean" (Lüders/Rauschenbach 2001: 566), es decir, se debe tomar en serio la re-vinculación de los temas de desarrollo y vida de los jóvenes a su contexto institucional escolar.

La perspectiva investigadora que asumiremos en este trabajo se basa, entonces, en la apropiación del trabajo social escolar en el contexto de "ser alumno", es decir, de la construcción social de una situación de vida estructurada a través de arreglos institucionales (vid. Oelerich 1996). El valor de la utilidad del trabajo social escolar es transmitido a los alumnos en primer lugar por medio de los expertos trabajadores sociales como portadores de los significados (o como medio de transmisión) de la oferta, en segundo lugar por medio de las experiencias de relación con estos profesionales en la interacción pedagógica cotidiana, en tercer lugar por medio del grado de compromiso de estas relaciones y en cuarto lugar por medio de la experimentación de que sus temas tienen relevancia y reciben el reconocimiento de terceros. Una aproximación investigadora orientada en los destinatarios permite reflejar determinadas conclusiones acerca de la relación de contenido entre la dimensión del uso y la dimensión de la utilidad (valor del uso). Esta conexión analítica de ambas dimensiones permite comprobar, en quinto lugar, la relevancia de la relación intergeneracional, articulada de modo pedagógico, como elemento estructural del trabajo social escolar.

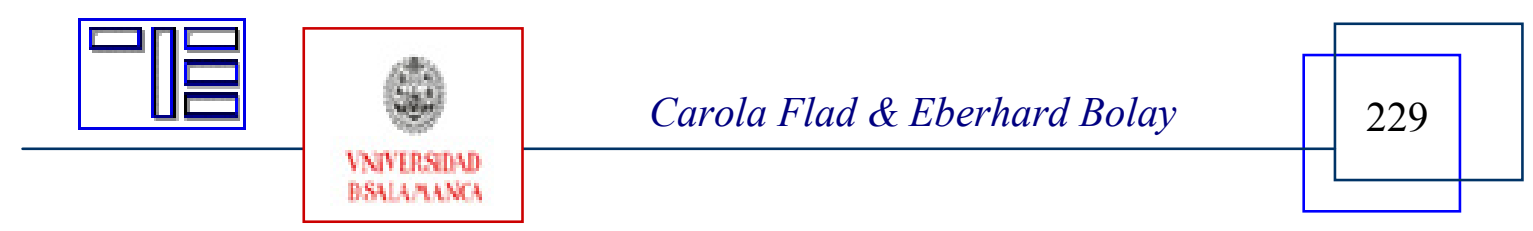


Revista Electrónica Teoría de la Educación.

Educación y Cultura en la Sociedad de la Información.

http://www.usal.es/teoriaeducacion

Vol. 9. No 2. Mayo 2008

Después de la introducción al contexto de investigación en el que se enmarca este trabajo, mostraremos en un ejemplo práctico el papel que el trabajo social escolar puede adquirir en la superación biográfica de situaciones problemáticas. En un segundo paso se desarrollará uno de los resultados esenciales de esta investigación. Este resultado está descrito como la "calidad de la experiencia "con el trabajo social escolar. Se analizarán componentes de esta calidad experimentada en la confrontación de lógica académica y arreglo institucional por un lado, y deducción y apropiación juvenil por otro. En un tercer paso se sistematizarán las experiencias de los jóvenes sujetos como experiencias de vinculación y la consecuente interpretación de estas, en un cuarto paso, como tres modos de una interacción generacional.

Nuestras propuestas se reducirán a un mínimo de los procesos comunicativos y de interacción entre los expertos y los sujetos juveniles. A modo conclusivo describiremos condiciones marco que favorecen al desarrollo de una relación generacional orquestada de manera pedagógica en el contexto del trabajo social escolar.

\section{2.- CONTEXTO DE INVESTIGACIÓN Y BASE DE DATOS.}

Un análisis que se enfrente a las dimensiones cualitativas del uso en el contexto de investigaciones empíricas acerca de la cooperación de apoyo juvenil y escuela, hasta el momento sólo ha sido articulado marginalmente (Bolay 2004b: 34).

En tres estudios de caso en el contexto del trabajo social escolar - como práctica de cooperación de mayor intensidad entre ayuda a los jóvenes y escuela - se han analizado también los tipos analíticos de los respectivos entornos de vida de aquellos a los que la oferta está dirigida primordialmente y a quienes se intenta apoyar: en el marco de un estudio de caso en una escuela del tipo Hauptschule ${ }^{2}$ (Bolay et al. 1999: 40s.); en un estudio regional (Bolay/Flad/Gutbrod 2003: 47s.) y en un análisis empírico del apoyo regional en el programa "Jugendsozialarbeit an Schulen in Baden-Württemberg" [trabajo social con jóvenes en escuelas de Baden-Wurtemberg] (Bolay/Flad/Gutbrod 2004: 236ff), cuyo material de datos y análisis están en el centro de lo expuesto a continuación. Se han realizado entrevistas individuales y de grupo de entre 40 y 120 minutos de duración con catorce alumnas y alumnos de entre 14 y 17 años de edad en cinco escuelas distintas (Hauptschulen y escuelas técnicas) ${ }^{3}$ Como instrumento de medición se ha utilizado un hilo conductor manejado de modo abierto y flexible, dependiendo del tiempo disponible y el transcurso de la entrevista.

Los jóvenes entrevistados dispusieron de este modo de la libertad de destacar los puntos claves por cuenta propia. Como ámbitos en los que se plantearon las preguntas estuvieron preestablecidos: el acceso a las ofertas del trabajo social escolar, la valoración de ese trabajo, la ubicación del trabajo social escolar en el entorno social e institucional de la escuela, la reconstrucción de la situación de vida comunicada por la escuela a los alumnos.

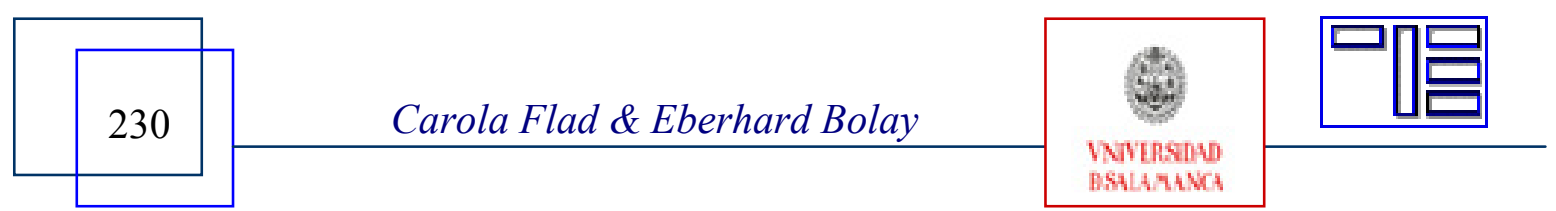




\section{3.- EL EJEMPLO DE GEN, QUINCE AÑOS DE EDAD Y ALUMNO EN UNA HAUPTSCHULE.}

Gen cursa el sexto grado en la Escuela A. ${ }^{4}$ Desde hace dos años y medio vive de forma permanente en Alemania, anteriormente había vivido en la República Dominicana, habiendo estado repetidamente y por algunos meses de visita en Weißstadt, acudiendo frecuentemente al centro para la juventud del lugar (Jugendhaus). Después de asumir la ciudadanía alemana visitó durante seis meses una escuela internacional de idiomas, para aprender alemán. Ahora lo habla de modo "gescheit" [en el dialecto local significa: bastante bien, n.d.t.], como lo describe él mismo. Los padres están divorciados, el padre reside en "América". Conoce al trabajador social desde un buen tiempo, ya desde sus visitas al centro juvenil Jugendhaus que se ha convertido en su segunda casa. "Estoy casi todos los días allí", especialmente los viernes, cuando es día de "estudio" y se graba música rap. Gen siente, piensa y actúa en su auto-identificación cultural juvenil como rapero. Los raperos son talentos del lenguaje, que por un lado pueden hablar muy bien $\mathrm{y}$, por otro, adquieren reconocimiento por su utilización creativa del idioma, subyugando las reglas gramaticales bajo el ritmo del respectivo beat. Si Gen da a entender que "se puede decir que somos raperos", esta expresión contiene un tono especial: justamente la virtuosidad del habla como medio central de expresión y núcleo identitario se le es negado en la escuela, y destacado como déficit, pues sus conocimientos del idioma no son suficientes para seguir el paso en la Escuela A. Por ello "antes nunca iba al Cole, porque estaba cagado de susto. Si no entendía las tareas o algo así, no iba por miedo a que los maestros me dijeran algo."

Gen se siente responsable por su madre. "Si algo se organiza, una excursión como ahora en las vacaciones de Pentecostés, casi nunca voy, porque mi Mum quedaría sola por dos semanas.(...) no sé, en realidad estoy acá para cuidar a mi Mum (...) no la dejo dos semanas sola (pausa). No sé. No hay confianza." Sus planes para el futuro también están influenciados por su relación con su madre. Quiere ser mecánico argumentando que "eso me gusta, conozco bien la materia. Siempre arreglo el auto de mi Mum. Porque, cuando era joven, mi padre tenía una cuestión de mecánico. Yo siempre lo acompañaba y me lo explicaba todo. Pero mi padre y mi Mum están divorciados. Por eso....ahora yo tengo que cuidarla."

Reparar automóviles es un momento familiar, en el que Gen asume las tareas del padre ausente y crea así un lazo con su pasado, en un momento de relación positiva padre-hijo y la vincula con el esfuerzo actual de cuidar sigilosamente a su madre. El deseo profesional del mecánico está relacionado, desde la experiencia de Gen, con una familia intacta. En el contexto de su familia, Gen asume un "papel alternativo" a su infancia, se auto diseña como el protector viril de su madre.

A diferencia de sus compañeros, Gen rechaza las descripciones compadre o conocido para caracterizar al trabajador social de la escuela. "Tobi es un adulto, es el único mayor en el que confío", "siempre lo llamo tío". La metáfora del tío incorpora al trabajador

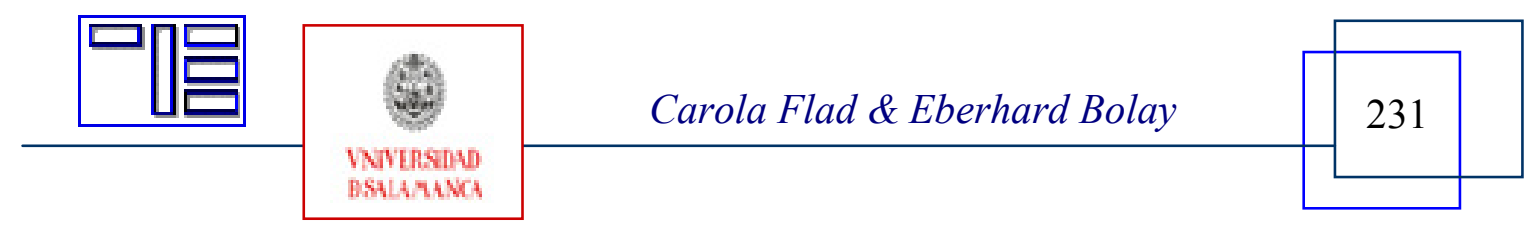


Revista Electrónica Teoría de la Educación.

Educación y Cultura en la Sociedad de la Información.

http://www.usal.es/teoriaeducacion

Vol. 9. No 2. Mayo 2008

social al contexto familiar inmediato. Lo que califica a Tobias Herder como "tío" - es decir, una persona adulta, que tiene un afecto natural para con él - se puede describir en tres aspectos:

- "Tobi "ha probado ser un compañero confiable. Debido a sus deficientes conocimientos del alemán, Gen aprovecha la posibilidad de dejarse ayudar por Tobias Herder: "se ha sentado día tras día, porque tuve que redactar el ensayo sobre hip-hop con palabras propias y eso no lo puedo tan bien, porque no hablo tan bien alemán. Y entonces me ayudó siempre y me explicaba qué significaba qué cosa. Al final salió una matrícula de honor." En su descripción del estudio conjunto resalta el énfasis en que "se ha sentado día tras día". Esta expresión no está vinculada al tiempo real, sino ha de entenderse de modo simbólico y destaca la insistencia y consecuencia con la que ambos persiguieron su meta de redactar un buen ensayo. La presentación del tema hip-hop se ha convertido desde el punto de vista subjetivo en un proyecto conjunto, en el cual el trabajador social le ha facilitado a Gen su manejo del lenguaje.

- Tobias Herder actúa en representación: Gen quiere ser mecánico. Al ser consultado si hablaría de esto con el trabajador social, responde, "claro, casi todos los días. El es el único que me ayuda en eso. Y el colegio y eso, con ello... Es el único que me deja ... que se toma el tiempo de ir conmigo a la escuela y habla con la gente y eso." También en esta parte resalta la descripción de una actitud y afecto percibidos como extraordinarios. Tobias Herder le ayuda a conseguir su meta profesional. Acompaña de manera responsable ese "proyecto", organiza para él un traslado de la Hauptschule a una clase en la formación profesional preparatoria, negociando en representación de la madre la aceptación en la escuela nueva.

- El trabajador social tiene presencia para Gen en una estructura duradera: A diferencia de su propio padre, Gen recibe afecto continuo y también crítico por parte del trabajador social. Tiene presencia en una estructura confiable y profesional. "Ya lleva algunos años en el Centro de Jóvenes. Trabaja allí, se podría decir, toda la vida." Con esta asunción exagerada, Gen justifica el carácter estable y duradero del apoyo. Interpreta su relación con Herder sobre el fundamento de una oferta vinculante en el contexto profesional del Centro de Jóvenes y extendida ahora hacia el entorno escolar.

Un quinceañero cansado de la escuela se convertido, pues, en un joven que percibe su formación como positiva y que, entre otras razones, ha cambiado debido a su relación con el trabajador social escolar. Desde ya cuatro años, es decir casi un tercio de su vida, experimenta un apoyo biográfico intenso aunque restringido. "Es distinto ahora. Ahora soy distinto." Sus problemas escolares han disminuido, ya no tiene problemas de asistencia y se prepara para las clases. Su respuesta a la pregunta si asiste con ganas a la escuela es diplomática: "Nadie va con ganas a la escuela, pero si hay que ir, haya que ir.

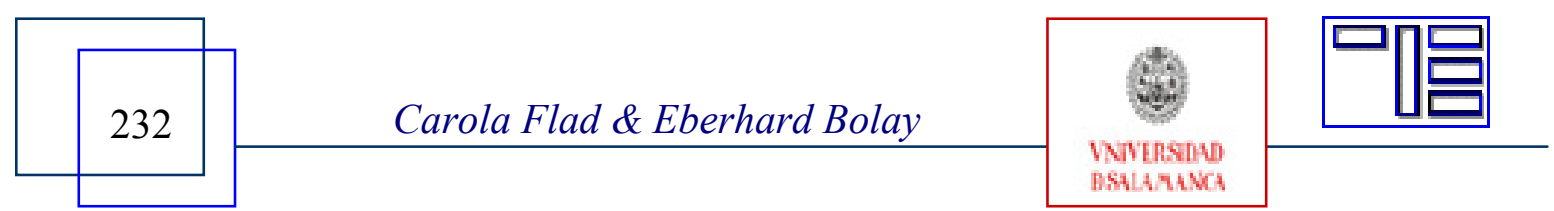


Esto lo he comprendido ahora." También su interacción con sus compañeros es menos conflictiva. "Ya no hay peleas. Antes me ponía muy nervioso por todo. Ahora ya no. "El trabajador social escolar apoya a Gen en tales cambios, fomentando su capacidad de pensar en alternativas a sus modelos habituales de superar determinados hechos.

En el caso de no asistir a la escuela se trataba de asumir con "mayor claridad" la relación entre el comportamiento "no asistir a la escuela" y su causa, "esquivar los problemas del idioma“. En su relación con Tobias Herder se normaliza su condición de joven. No es el rapero que llama la atención como provocador, peleador o por boicotear la escuela y tampoco sólo el adolescente quinceañero que asume toda la responsabilidad emocional para su madre. Es un adolescente al que se trata de acuerdo a su edad y a sabiendas que quiere encontrar una perspectiva de desarrollo particular desde la fijación en el presente hacia un posible futuro.

En resumidas cuentas, este ejemplo ilustra una serie de cosas:

- Ha sido escogido como un ejemplo típico para la apropiación del trabajo social escolar por parte de alumnos, puesto que con ello queda en evidencia que los jóvenes aprovechan la oferta de múltiples modos, no sólo por una única razón permanente.

- Desde la perspectiva de Gen, la oferta del trabajo social escolar parece modelada a sus necesidades, puede acudir a ella en las cuestiones determinantes. Así, además de la perspectiva de continuidad adquiere relevancia biográfica para el joven.

- El ejemplo demuestra además que los usuarios juveniles del trabajo social escolar consideran la oferta como otra opción de relacionarse, que procesan de forma nueva y distinta en el contexto de la experiencia escolar como relación intergeneracional. Perciben su relación con los trabajadores sociales como distinta a los tipos de relación habituales en el contexto escolar, entre profesorado y alumnado (como "relación generacional intergeneracional") o entre compañeros (como "relación generacional intrageneracional", (vid. Lüscher/Liegle 2003: 172).

- Con la estabilidad del joven en su función como alumno queda en evidencia la utilidad inmediata del trabajo social escolar, así como en su habilitación en la manera de interactuar con las exigencias estructurales y de comportamiento en la escuela.

- La perspectiva de la lógica estructural en la materia, que guía desde el punto de vista profesional la orientación del andamiaje institucional y que determina la posición profesional propia del educador, no tiene relevancia para los alumnos. Lo dicho por Gen muestra una perspectiva distinta, la de un trabajador social

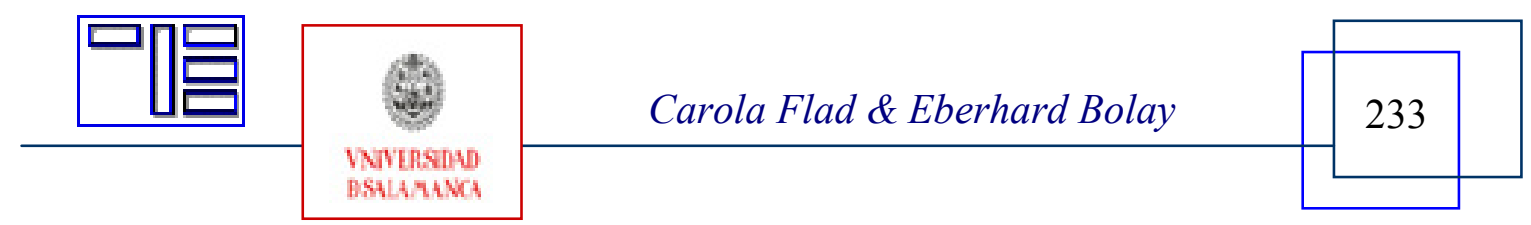


Revista Electrónica Teoría de la Educación.

Educación y Cultura en la Sociedad de la Información.

http://www.usal.es/teoriaeducacion

Vol. 9. No 2. Mayo 2008

escolar con disponibilidad ilimitada. Esta diferencia de perspectivas se puede descifrar como una selección de una multitud de opciones de formas específicas de apoyo y acompañamiento personal, debida a sus propias necesidades. Esto se puede ejemplificar con dos casos. En razón a los puntos de referencia "tiempo" y "oferta" se contrasta el cálculo sustancial-conceptual con el cálculo de utilidad del joven.

\section{4.-ARREGLO SUSTANCIAL-CONCEPTUAL Y USO JUVENIL COMO PERSPECTIVAS DISTINTAS.}

Tiempo es desde la perspectiva profesional un factor determinante que influye en la elaboración de la oferta de apoyo a los jóvenes. La consulta estandarizada de profesionales demuestra que para la conceptualización de una oferta, aparte de las necesidades definibles del alumnado y de las líneas guía de su aproximación profesional, los recursos de tiempo tienen que ser considerados como determinantes (vid. Bolay/Flad/Gutbrod 2004: 67s.). Pero el tiempo no solamente limita como valor calculatorio el espectro y la intensidad de logros y formas de cooperación. Como factor subjetivo el 'tiempo limitado' -especialmente en el caso de puestos parciales- se expresa a largo plazo, con insatisfacción y carga en la actividad cotidiana del profesional (vid. Bolay/Flad/Gutbrod 2003: 44). El tiempo ha de ser administrado, por ende, en la lógica estructural profesional de tal modo que no implica pérdidas en la implementación de la planificación conceptual.

Los usuarios juveniles describen experiencias completamente distintas en relación al aspecto del tiempo. Al mismo tiempo las experiencias temporales adquieren un papel esencial en la apropiación de la oferta de apoyo juvenil. Contrastando con la estructura temporal escolar definida, la que los alumnos están obligados a asumir, experimentan en las ofertas del trabajo social escolar un potencial de estructura temporal flexible y acomodable de acuerdo a las necesidades y que ellos mismos pueden manejar. Así se sienten tomados en serio por parte de los trabajadores sociales. En el caso ejemplar de Gen ya hemos podido comprobar la relevancia del factor tiempo y la connotación del afecto especial percibido en ese sentido por él. Esta calidad de la percepción de valores temporales aparentemente no regulados se repite tipificadamente en las declaraciones de los jóvenes: "uno siempre puede ir", "siempre están dispuestos a hablar con uno, para eso están", "si tengo problemas incluso llamo al móvil", etc. El tiempo también es un criterio mediante el cual evalúan la oferta de la ayuda juvenil: "lo peor sería que un alumno entrase y él dijera, 'no tengo tiempo".

Planificar la oferta y el contenido de la oferta de acuerdo a las necesidades, implementar esos planes y evaluar la implementación en razón a posibilidades de optimización son los retos profesionales esenciales del trabajo social escolar. Unidades de trabajo tienen que ser comparados y se tienen que definir prioridades, ya que no se pueden crear ofertas de manera ilimitada. Aparte de la capacidad de tiempo los

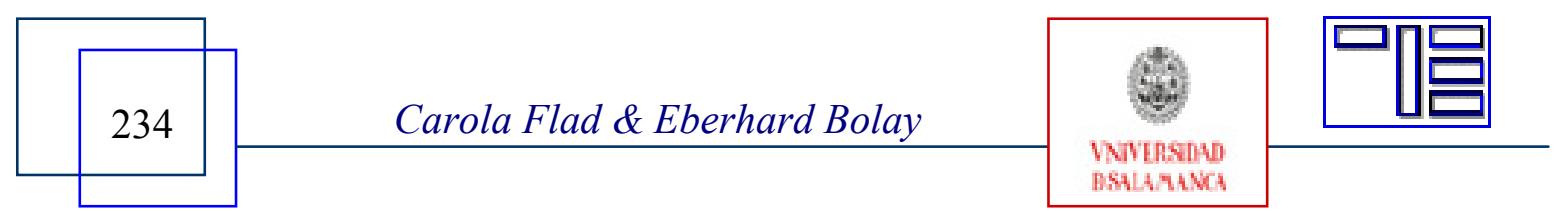


conocimientos y habilidades propios son importantes, al igual que equiparar las ofertas de grupo por un lado y apoyos individuales por otro, el acoplamiento con ofertas existentes en la escuela y la posibilidad de cooperación con instituciones extraescolares.

La organización sustancial de las ofertas no es de mayor relevancia para los alumnos. $\mathrm{Su}$ acceso a las respectivas ofertas se caracteriza más bien por un desarrollo que va más allá del uso concreto de una determinada oferta hacia una forma de contacto más allá de lo especializado. Un chico lo describe así: "Primero ir y decir 'quiero redactar una aplicación, ¿me ayudan ustedes? Llegar al punto así y después ya más en lo personal, conversar, tomar un café." En el centro está aquella persona que se consagra como la persona adulta de referencia y que posteriormente incorporan a sus necesidades. "Como que me atrae conversar con ella" (dice una alumna). El análisis de la experiencia de los alumnos demuestra que su actitud de apropiación indica más allá de una simple utilización de un fragmento de la oferta, mostrando contextos propios de sentido particular: aprender en una relación intergeneracional de manera autodeterminada, "pasarla bien" en el significado del reconocimiento vivido de la madurez propia, o cultivar un lugar en la escuela en el que se pueden mezclar intereses funcionales como la redacción de una aplicación, con intereses del entorno de vida. Estos intereses y necesidades son integradores como sub-textos en el uso de las ofertas.

La oferta de apoyo juvenil está presente y familiar en los jóvenes en un sentido estructural que destaca, en el ámbito profesional en el que se realiza, los momentos auténticos de una relación "natural" y no una relación funcional. El trabajo social escolar es para los alumnos más que una serie de ofertas más o menos atractivas. En lo que sigue revisaremos más detenidamente aquellos aspectos, de acuerdo a los que los jóvenes evalúan a los trabajadores sociales y que implican, a la vez, características de esa opción de relaciones.

\section{5.- EL TRABAJO SOCIAL ESCOLAR COMO OPCIÓN EXTENDIDA DE RELACIÓN.}

Las altas expectativas morales hacia los profesionales demuestran la importancia del trabajo social escolar como opción en la red de relaciones en la escuela. "Si ellos prometen algo, también lo han de cumplir, nosotros confiamos en ellos." A la vez, la imagen que los jóvenes describen del trabajo social escolar indica un alto grado de compromiso. "Ellos quieren lo bueno para uno", "también pelea para que les vaya mejor a los alumnos", "no cesan en sus esfuerzos, porque quieren sacar a los jóvenes de abajo y convertirlos en hombres y mujeres que puedan trabajar en un futuro". Por otro lado, el profesorado es medido con la vara algo más baja. Aunque el compromiso particular de algún maestro es destacado como apoyo especial, el carácter obligatorio de las clases y las expectativas de las funciones determinan la interacción. "Los profesores están ahí solo para dar clases." Los alumnos expresan una conexión entre la buena reputación (y

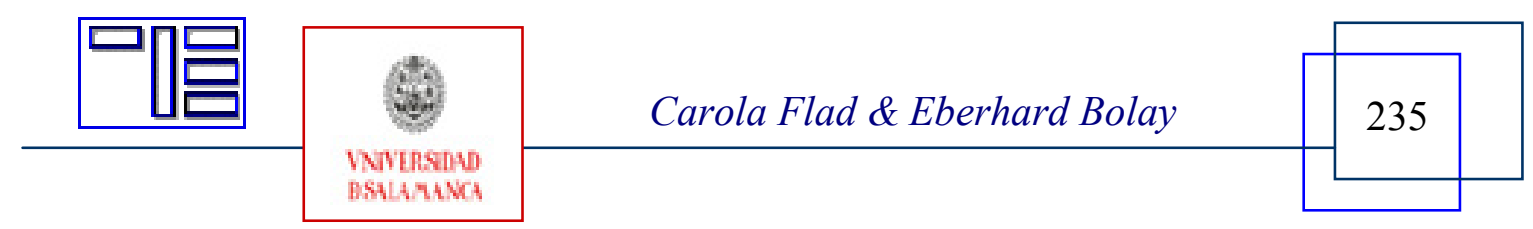


Revista Electrónica Teoría de la Educación.

Educación y Cultura en la Sociedad de la Información.

http://www.usal.es/teoriaeducacion

Vol. 9. No 2. Mayo 2008

la popularidad de los expertos sociales entre los jóvenes) y determinadas estrategias de acción e interacción del trabajo social escolar. Esto ha de ser profundizado en cuatro puntos.

\section{Accesibilidad.}

El hecho de que los jóvenes acudan al trabajo social escolar ansiosamente se debe también a que los trabajadores buscan el contacto con ellos y lo cuidan. Los jóvenes consultados describen múltiples aspectos de una accesibilidad arreglada concientemente por los profesionales. Destacan que reparten su afecto de manera justa y no seleccionan entre los jóvenes. "Tan normal como uno es nos aceptan". Que este contacto con antiguos alumnos puede seguir existiendo y no está limitado funcionalmente a la etapa escolar simboliza una relación auténtica. "Se mantiene el contacto, diría yo"- o dicho de otro modo: son contactos confiables biográficamente acompañantes.

\section{Sensibilidad hacia cultura juvenil.}

Una parte de los jóvenes consultados describe el contacto con los expertos del apoyo juvenil como basado en las reglas de la cultura juvenil. "Habla con nosotros como nosotros hablamos, para que vaya mejor." Este habito profesional vinculable a la cultura juvenil les permite a los adolescentes, aparentemente, la posibilidad de incorporar a los profesionales en su horizonte de experiencias. Interpretan esos módulos de interacción y comunicación como competencia profesional, es decir, no confunden a los trabajadores sociales con compañeros de la misma edad. "En realidad analiza a los jóvenes todo el día (...). Siempre está con jóvenes. Por eso sabe lo que es bueno para ellos y como hablarles". Los jóvenes lo entienden como especificidad de los trabajadores sociales, adquirir conocimiento acerca de los jóvenes y aplicar ese conocimiento en su oferta.

El conocimiento de los profesionales acerca de la cultura juvenil es destacado por los jóvenes como elemental en dos sentidos. Primero porque trasciende hacia las ofertas que se establecen con orientación en ellos. Por otro lado se refleja en el hábito profesional y autentifica a los trabajadores sociales como personas importantes de referencia. Con esto está vinculado otro componente del significado en la experiencia de los jóvenes que no sólo se explica con la experiencia de una proximidad de cultura juvenil. A diferencia del profesorado no tienen que recurrir a la diferencia de edad para justificar una relación jerárquica. "Nos tratan como amigos, no como alumnos o niños chicos, sólo por ser mayores. Uno puede hablar mucho mejor con ellos". El reconocimiento armonizador de experiencia y competencia de vida que se demuestra en esa experiencia ha de ser sistematizado a continuación como expresión de la conformación de una relación basada en la negociación.

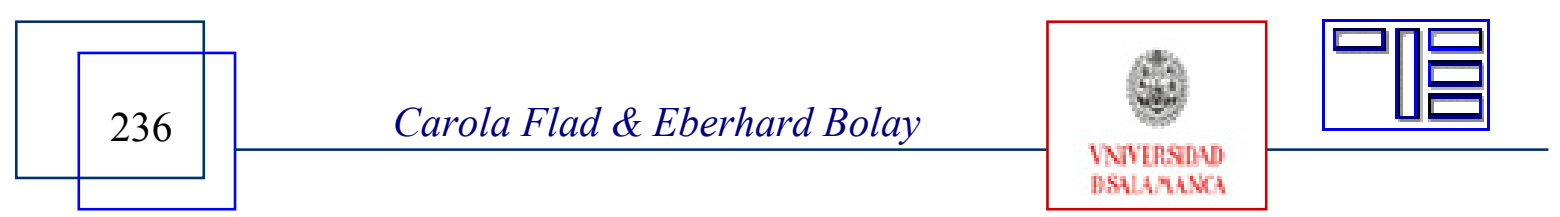




\section{Conformación de relaciones en la negociación.}

Los jóvenes interpretan la oferta de relación concedida por el trabajo social escolar como algo distinto al orden caracterizado por la formalidad entre profesorado y alumnos. "Profes son más bien estrictos, porque quieren que uno aprenda algo". La relación con los trabajadores sociales fortalece la competencia de valoración y decisión de los jóvenes. La relación con sus maestros es considerada bajo la premisa de la evaluación y recriminación. Es difícil negociar con maestros que "otorgan partes", más bien hay que cuidarse en lo que se refiere a expresar la opinión propia.

El trabajo social escolar como instancia casi librada de sanciones escolares sienta las bases para una interacción equivalente. "Aunque uno le diga algo en su cara no hacen anotaciones o algo. Más bien preguntan acerca del por qué“. El ejemplo de la golpiza en el recreo es un ejemplo recurrente entre los alumnos para explicar los distintos roles. "Si por ejemplo dos se pelean en el patio, viene el profesor y dice que tienen que copiar el reglamento escolar. Si pasa uno de los trabajadores sociales, los separa y pregunta por qué pelean y cómo se puede solucionar. Son muy distintos“. Los alumnos comprueban esa opción de relación basada en la negociación y no en la manutención del orden ("reglamento") en distintas ocasiones.

Desde una perspectiva formal la obligación de los profesionales de guardar silencio es una base de confianza; en relación al trato pedagógico los jóvenes estiman un estilo no paternalista en secuencias cotidianas y la comunicación dialogante sobre un encargo a cumplir.

\section{Conocimientos transferibles de adultos.}

A través de los trabajadores sociales se les brinda a los jóvenes un conocimiento específico que interpretan como ventaja positiva que ha de ser aprovechada. "También aprendemos de nuestros errores. Ellos nos lo explican“. La alumna aprende a reflejar su propio comportamiento y lo comprende como momento de aprendizaje. Colateralmente, sin estructuras previas y reiteradamente se dan estas posibilidades de aprendizaje. Ella parte de la base que los mundos de experiencia entre joven y profesional no están demasiado separadas, "somos como amigas". De todos modos está conciente de la diferencia de edad: "Tienen más experiencia, Seguramente han vivido cosas, y bueno, ahora nos las pueden transmitir."

La valoración del conocimiento adulto está relacionada con aspectos genéricamente específicos en el uso del trabajo social. Si los jóvenes pueden escoger si entrar en confianza con un trabajador o una trabajadora social, habitualmente escogen aquel del mismo sexo. Para esa decisión parece ser determinante, que la persona dispone de conocimientos específicamente de chica o de chico debido a su propia socialización, por lo que podría dar consejos más adecuados.

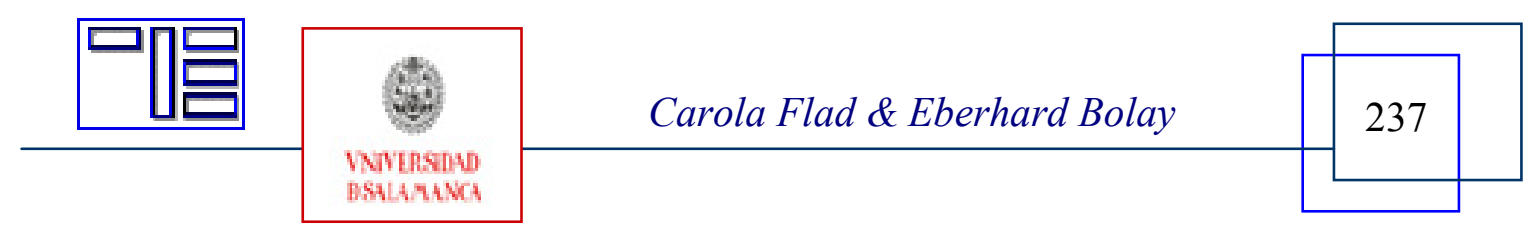


Revista Electrónica Teoría de la Educación.

Educación y Cultura en la Sociedad de la Información.

http://www.usal.es/teoriaeducacion

Vol. 9. No 2. Mayo 2008

En resumidas cuentas queda demostrado que los alumnos valoran a los trabajadores sociales en las escuelas como adultos estables y confiables, a los que pueden acceder como alternativas a las vinculaciones preestablecidas $\mathrm{y}$, en parte, pedagógicamente perturbadas en clases o incluso las familias.

\section{6.- CONCEPTOS DE INTERPRETACIÓN EN EL MARCO DE LA RELACIÓN PEDAGÓGICA INTERGENERACIONAL.}

El análisis de las relaciones comunicativas y de interacción entre jóvenes y profesionales de la ayuda juvenil en la escuela indica a interpretarlas como espacio y procesos de aprendizaje, cuya base está en una relación intergeneracional productiva. EL grado de compromiso, la constancia y reciprocidad son indicadores de calidad, según Lüscher y Liegle con base en resultados sobre la teoría de vínculos, que influyen en el aprendizaje intergeneracional en la relación padres-hijos (2003: 187f.). En el ejemplo inicial se ha podido comprobar como el alumno Gen, ante el trasfondo de relaciones familiares inestables experimentadas, (re)encuentra en su relación con el trabajador social una constelación tipo supletoria para el afecto paternal. Al evocar al trabajador social como persona de referencia casi familiar, describe a la vez cualidades características para las experiencias en una relación intergeneracional y que abren ámbitos de aprendizaje con carácter formativo para la personalidad en el marco de tales relaciones.

El análisis demuestra, además, que los jóvenes aprovechan de manera productiva las tensiones y ambivalencias inherentes en esta forma de relaciones (cfr. ibíd. 2003: 285s.): como "otros adultos", en el arreglo del "aprendizaje informal" y en el hábito profesional específico, orientado en la reciprocidad del aprendizaje. A continuación se profundizará en estos tres aspectos.

\section{“Otros" adultos en la percepción de los jóvenes y su necesidad ambivalente}

Un primer punto de referencia se deriva del concepto del "otro adulto" (Wolf 2002; para la fundamentación pedagógica juvenil vid. Böhnisch 1998, 1998b), que recoge varias facetas en la busca de identidad de los adolescentes - su necesidad, así como la adquisición de madurez y competencias de vida. En la reestructuración psíquico-social de identidad en la juventud (pubertad) los adolescentes tienen que encontrar reiteradamente el punto medio entre experiencia y sentimientos, en los que se perciben más bien dependientes, y el otro polo, el deseo y la obligación social de autonomía. Los adolescentes se remiten especialmente a su peer-group, pero también requieren de adultos que les sirvan de apoyo y punto de enfrentamiento. La terminología del "otro adulto" se refiere a "adultos específicos que, por un lado, apoyan los intentos de los jóvenes de reclamar su autonomía, pero que los perciben, por otro lado, también en sus necesidades"(Wolf 2002: 219). ¿Qué niños y jóvenes se encuentran con adultos en la escuela que cumplen con estas condiciones? Los maestros están en una relación

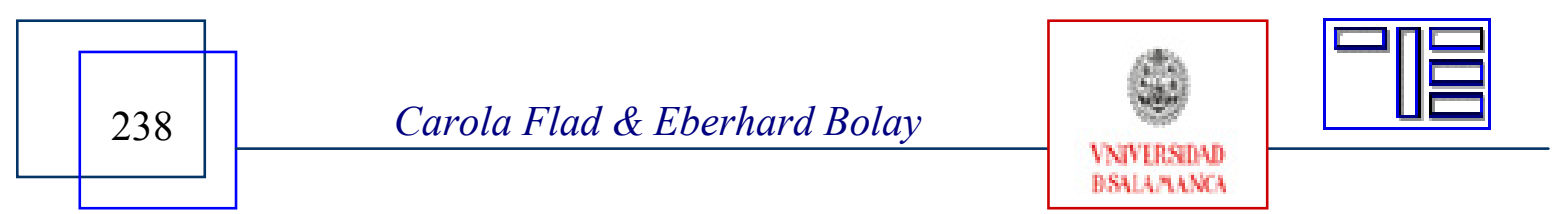


ambivalente: debido a su función no pueden sobreponerse mucho a la tarea de la transmisión de conocimiento y evaluación del rendimiento y presentarse como "otros adultos". Trabajadores sociales sensibilizados para la pedagogía juvenil, en cambio, pueden asumir el papel de "otros adultos" con mayor facilidad, porque su tares dirigida al apoyo y la ayuda lo permite, o más bien lo exige.

Las referencias personales al trabajo social escolar están caracterizadas por el principio de la conformación de relaciones a través de negociaciones. Se trata de una relación definida a través de espacios de acción. Estos espacios permiten mostrarse en un tono más personal y aportar de múltiples maneras. Con ello se ha marcado una relación intergeneracional que se incorpora conscientemente como un campo de prueba en la transición del ser joven al mundo adulto. Especialmente las atribuciones personales en la interpretación de los alumnos ilustran que los adolescentes buscan en los trabajadores sociales "adultos interesados, que las chicas y los chicos pueden orientar en su percepción de empujarse hacia el mundo adulto y a la vez - a diferencia de la relación con padres o maestros- referirse a su juventud" (Böhnisch 1998a: 34).

\section{Aprendizaje informal en la relación intergeneracional.}

Un segundo punto de referencia de la relación intergeneracional deriva del enfoque en la creciente relevancia de "experiencias en la vida" (Thiersch 2002: 67), cuyos momentos informales de formación aportan a la "conformación de vida como superación de vida" (ibíd.: 60). Conceptos de formación y prácticas de formación también del Trabajo Social están sumergidos a una reorientación. Más allá de formas de aprendizaje puestas en escena o planificadas, ambos campos de acción pedagógica deben crear una "relacionabilidad" con espacios no preestablecidos y libros de experimentación e interpretación de esas experiencias. ${ }^{5}$ Porque "el principio del autoaprendizaje se carga de tareas de conformación de vida al abierto y se convierte en el momento central del manejo en el proceso formativo. Disposiciones vinculantes pasan a un segundo plano, ocasiones y recomendaciones aumentan en importancia" (ibíd.: 66). Con la referencia a Thiersch queda en evidencia la relevancia de estructuras de ocasiones y posibilidades del autoaprendizaje, que no se desenvuelven en un entorno planificado de formación, sino en un sentido descriptible como formación por actos propios.

El trabajo social escolar posibilita procesos formativos específicos mediante competencias de acción relativas a situaciones diversas. Estos son distintos a los arreglos escolares formales. El trabajo social escenifica procedimientos de la formación informal y abre un terreno para procesos informales de aprendizaje, puesto que los trabajadores sociales crean posibilidades para los alumnos de experimentar situaciones cotidianas y trabajar en solucionarlos. ${ }^{6}$ Los resultados empíricos de las investigaciones indican que desde la perspectiva de los jóvenes, los trabajadores están perfectamente habilitados para esta tarea si no recurren a modos formales de comunicación e

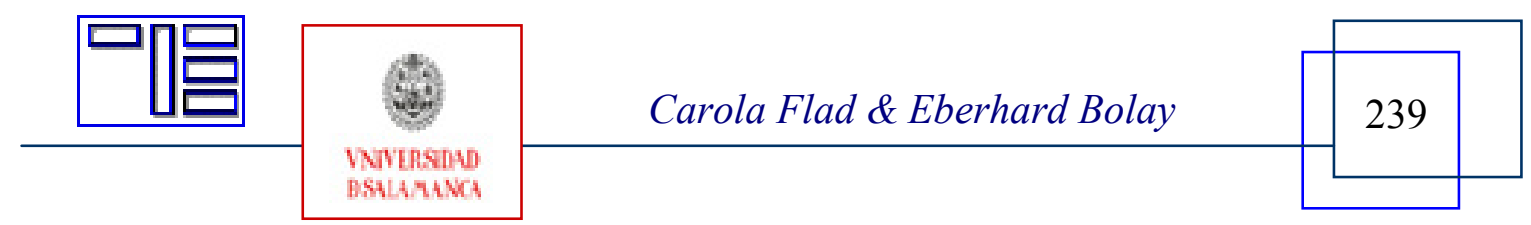


Revista Electrónica Teoría de la Educación.

Educación y Cultura en la Sociedad de la Información.

http://www.usal.es/teoriaeducacion

Vol. 9. No 2. Mayo 2008

interacción. Se convierten en ídolos secretos en actuar solidariamente, en argumentar y en defender una posición propia.

\section{Un hábito sustancial orientado en la reciprocidad del aprendizaje.}

Un tercer punto de referencia deriva con miras en las reflexiones de Lüscher y Liegle. El punto de partida es una constante antropológica, "que la práctica social de las relaciones intergeneracionales (...) está vinculada intrínsecamente con el aprendizaje" (2003: 171). El aprendizaje intergeneracional ha de ser entendido bajo el aspecto de la reciprocidad. Esto se debe a que diferencias generacionales se relativizan bajo la influencia de cambios sociales bruscos, por lo que la donación unidimensional de conocimiento adquirido de la generación mayor a la menor es reemplazada por un aprendizaje mutuo entre las generaciones. "La generación genealógicamente joven actúa ante la generación media y mayor como generación 'transmisores debido a sus competencias tecnológicas y mediáticas, mientras que en otros sentidos - como por ejemplo en el financiamiento de la vida o en la función de alumno - sigue dependiendo de los mayores, reclamando la autonomía de la manera de vivir" (ibíd.: 176). Los adultos también aprenden, pues, en la formación de un adolescente, son a la vez transmisores de una "herencia cultural" como aprendices de la cotidianeidad conjunta (ibíd.: 177s.).

En una relación pedagógica configurada de modo profesional, sensible hacia un modo de vivir determinado por la cultura juvenil y su autonomía, esta correspondencia está incluida. Los jóvenes consultados describen de qué modo los trabajadores sociales aprenderían de los jóvenes y consagran de ese modo el valor propio de las prácticas juveniles: "Estudia a los jóvenes durante todo el día y sabe lo que es bueno para ellos". Aquí se puede observar un orden específico: Que los adolescentes aprendan de trabajadores sociales adultos depende que previamente ellos aprendan de los jóvenes. Se apropian, para decirlo de algún modo, del material del entorno de vida de los jóvenes y su aplicación positiva, para posibilitar ocasiones de aprendizaje, apoyo $y$ acompañamiento. Es importante resaltar en este contexto que los trabajadores sociales escolares están libres de la obligación de actuar como genios universales, lo que en principio imposibilita la reciprocidad. ${ }^{7}$

\section{7.-COMENTARIO FINAL.}

La meta de este aporte ha sido remitir la atención a los modelos de apropiación biográfica y uso del trabajo social escolar por parte de los alumnos. Podemos constatar que este enfoque investigativo puede ilustrar la relevancia de relaciones intergeneracionales paras los usuarios jóvenes y comprenderlas como componente estructural del trabajo social escolar. Se pueden concluir cuatro condiciones marco para la configuración de esta relación intergeneracional puesta en escena de manera pedagógica: En primer lugar una oferta fácil de alcanzar, estable y calculable para los

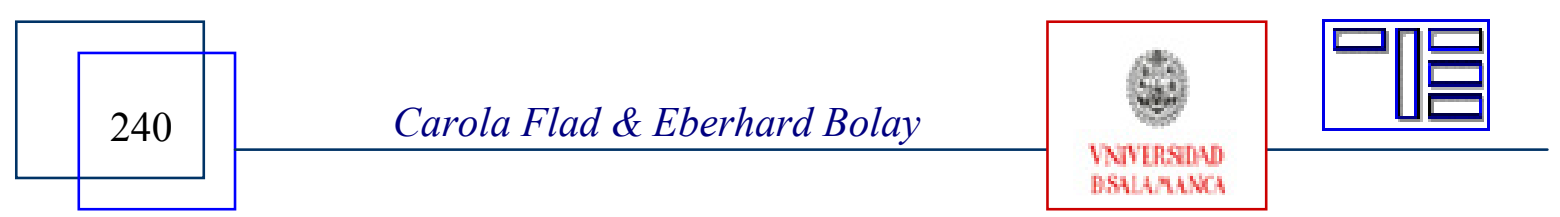


alumnos. Este ha de ser sensible para las necesidades y los intereses distintos de los jóvenes, ha de dirigirse en forma activa a ellos y ha de animarlos a experimentar la extensión de sus posibilidades de actuar. En segundo lugar el comportamiento relacional significa una presencia duradera y calculable del trabajador social en la escuela para posibilitar un valor curricular de su aprovechamiento. En tercer lugar los trabajadores sociales tienen una misión específica en las escuelas, posibilidades específicas de actividades y un repertorio sustancial determinado, distintos a las herramientas de la escuela. Esta experiencia diferente permite una extensión de procesos de socialización y formación de los jóvenes en el contexto del trabajo social escolar que tiene impacto positivo en el trato de los jóvenes hacia los imperativos escolares de acción. En cuarto lugar y asumiendo la perspectiva de los alumnos, el perfil profesional exigido de los trabajadores sociales es incompatible con una idea administrativa, consultiva-terapéutica o institucional-normativa de la actividad (Handlung). Parece más adecuado un tipo de profesión reflexivo participativo, enfocado en una "igualdad del dominio de conocimiento y experimentación tanto por parte de los profesionales como por parte de los destinatarios del trabajo social en situaciones concretas de actividad" (Kunstreich/Lindenberg 2002: 358).

\section{8.- BIBLIOGRAFÍA}

BETTMER, F./MAYKUS, S./HARTNUSS, B./PRÜSS, F. (2002). Theoretische und empirische Klärungen zum Verhältnis von Jugendhilfe und Schule. Archiv für Wissenschaft und Praxis der Sozialen Arbeit, 2 (33), 12-42.

BÖHNISCH, L. (1998). Der andere Blick auf die Geschichte. Jugendarbeit als Ort der Identitätsfindung und der jugendgemäßen Suche nach sozialer Integration. En BÖHNISCH, L./RUDOLPH, M./WOLF, B. (Eds.): Jugendarbeit als Lebensort. Jugendpädagogische Orientierungen zwischen Offenheit und Halt, (pp. 19-37). Weinheim/München: Juventa.

BÖHNISCH, L. (1998b). Grundbegriffe einer Jugendarbeit als ,Lebensort'. Bedürftigkeit, Pädagogischer Bezug und Milieubildung. En BÖHNISCH, L./RUDOLPH, M./WOLF, B. (Eds.): Jugendarbeit als Lebensort. Jugendpädagogische Orientierungen zwischen Offenheit und Halt, (pp. 155-168). Weinheim/München: Juventa.

BOLAY, E. (2004). Überlegungen zu einer lebensweltorientierten Schulsozialarbeit. En: GRUNDWALD, K./ THIERSCH, H. (Eds.): Praxis Lebensweltorientierter Sozialer Arbeit. Handlungszugänge und Methoden in unterschiedlichen Arbeitsfeldern, (pp. 147-162). Weinheim/München: Juventa.

BOLAY, E. (2004b). Kooperation von Jugendhilfe und Schule. Forschungsstand und Forschungsbedarf. Archiv für Wissenschaft und Praxis der sozialen Arbeit, $35,(2), 18-39$.

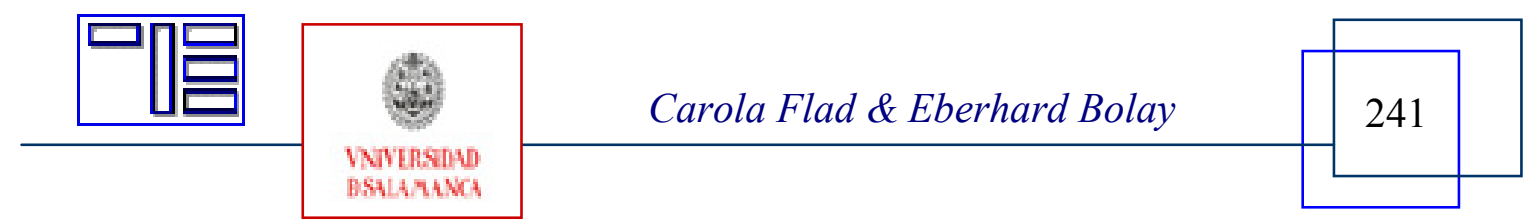


Revista Electrónica Teoría de la Educación.

Educación y Cultura en la Sociedad de la Información.

http://www.usal.es/teoriaeducacion

Vol. 9. No 2. Mayo 2008

BOLAY, E. ET AL. (1999). Unterstützen, vernetzen, gestalten. Eine Fallstudie zur Schulsozialarbeit. Stuttgart: Landeswohlfahrtsverband WürttembergHohenzollern.

BOLAY, E./FLAD, C./GUTBROD, H. (2003). Sozialraumverankerte Schulsozialarbeit. Eine empirische Studie zur Kooperation von Jugendhilfe und Schule. Stuttgart: Landeswohlfahrtsverband Württemberg-Hohenzollern.

BOLAY,E./FLAD,C./GUTBROD, H. (2004). Jugendsozialarbeit an Hauptschulen und im BVJ in Baden-Württemberg. Abschlussbericht der Begleitforschung zur Landesförderung. Tübingen/Stuttgart: Sozialministerium des Landes BadenWürttemberg.

DOHMEN, G. (2001). Das informelle Lernen. Die intentionale Erschließung einer bisher vernachlässigten Grundform menschlichen Lernens für das lebenslange Lernen aller. Bonn: Bundesministerium für Bildung und Forschung.

KUNSTREICH, T./LINDENBERG, M. (2002). Die Tantalus-Situation - Soziale Arbeit mit Ausgegrenzten. En THOLE, W. (Edt.): Grundriss Soziale Arbeit, (pp. 349366). Opladen: Leske + Budrich.

LÜDERS，C./RAUSCHENBACH，T. (2001). Forschung: sozialpädagogische. En OTTO, H./THIERSCH, H. (EDIT.): Handbuch Sozialarbeit/Sozialpädagogik, (pp. 562-575). Neuwied/Kriftel: Luchterhand Verlag.

LÜSCHER, K./LIEGLE, L. (2003). Generationenbeziehungen in Familie und Gesellschaft. Konstanz: UVK Verlag

MAYKUS, S. (2001). Schulalltagsorientierte Sozialpädagogik. Begründung und Konzeptualisierung schulbezogener Angebote der Jugendhilfe. Frankfurt/M.: Peter Lang Verlag

OELERICH, G. (1996). Jugendhilfe und Schule: Zur Systematisierung der Debatte. En FLÖSSER, G./OTTO, H.-U./TILLMANN, K.-J. (Eds.): Schule und Jugendhilfe: Neuorientierung im deutsch-deutschen Übergang, (pp. 222 - 237). Opladen: Leske + Budrich.

OELERICH, G. (2002). Schulsozialarbeit an Bielefelder Haupt- und Sonderschulen Abschlußbericht der Wissenschaftlichen Begleitung des Kooperationsprojekts Schulsozialarbeit, Bielefeld/Wuppertal (manuscrito sin publicar).

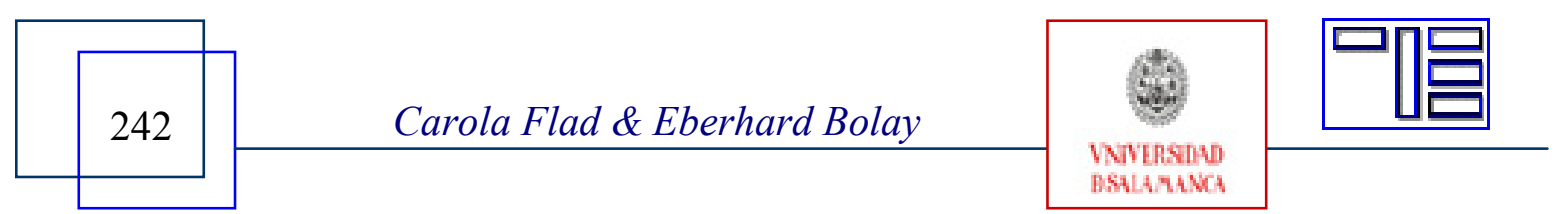


OLK, TH./BATHKE, G.-W./HARTNUSS, B. (2000). Jugendhilfe und Schule. Empirische Befunde und theoretische Reflexionen zur Schulsozialarbeit. Weinheim/München: Juventa.

RÖLL, F. J. (2002). Lernen in der Wissensgesellschaft. Wie Lehrende zu Navigatoren des Lernprozesses werden. Medien Praktisch, 26, (2), 17-21.

STREBLOW, C. (2003). „Ich war den ganzen Tag in der Schulstation“. Wildes Lernen in einem Schulsozialarbeitsprojekt mit schulmüden Jugendlichen. Sozial Extra, 27. $(2 / 3), 21-26$.

THIERSCH, H. (2002). Bildung - alte und neue Aufgaben der Sozialen Arbeit. En MÜNCHMEIER, R./ OTTO, H.-U./RABE-KLEBERG, U. (Eds.): Bildung und Lebenskompetenz. Kinder- und Jugendhilfe vor neuen Aufgaben, (pp. 57-71). Opladen: Leske + Budrich.

WOLF, B. (2002). „Andere“ Erwachsene. En: ARNOLD, H./SCHILLE, H.-J. (Eds.): Praxishandbuch Drogen und Drogenprävention. Handlungsfelder Handlungskonzepte - Praxisschritte, (pp. 219-234). Weinheim/ München: Juventa

\section{Notas:}

${ }^{1}$ Traducido por Michael Klode con el apoyo de la Vereinigung der Freunde der Universität Tübingen (Universitätsbund e.V.) / Alemania.

${ }^{2}$ [La estructura del sistema escolar alemán es -a grandes rasgos- la siguiente: después de cuatro años de formación básica (Grundschule) los alumnos acceden a distintos tipos de escuela, dependiendo de sus éxitos escolares: Hauptschule desde quinto hasta noveno grado, después de lo cual se accede a una formación profesional o una escuela preparatoria de formación profesional (Berufsschule) ; Gesamtschule desde quinto hasta noveno, décimo grado o graduación que mantiene abierto el futuro curricular; Gymnasium desde quinto hasta décimosegundo / décimotercer grado, es decir, hasta el examen final estatal que habilita para el acceso a universidades y formación continua. La terminología alemana es usada en este texto, nota del traductor. ]

3 Para los criterios del Sampling y el procedimiento concreto remitirse a Bolay / Flad / Gutbrod (2004:328).Por razones pragmáticas de investigación hemos enfocado para las entrevistas en alumnos que han experimentado el trabajo social escolar durante un largo tiempo y que lo perciben como algo positivo. Por supuesto que esto reduce la validez de resultados, porque no ilustran razones por las que jóvenes no asisten a las ofertas socioeducativas. Esta es una cuestión de gran relevancia, como lo demuestran los resultados de Olerich. En su investigación representativa del trabajo social en Hauptschulen y colegios especiales [Sonderschulen] en Bielefeld se pudo comprobar que para casi un tercio de los alumnos la asistencia al trabajo social escolar tiene carácter punitivo (Oelerich 2002:10).

4 La Escuela $A^{*}$ es un centro educativo y uno de los más grandes en Weissstadt con apróx. 1200 alumnos, 120 profesores y en promedio, 45 clases/cursos. En la Escuela A el trabajo social escolar ha sido incorporado desde 1980, por lo que es una de las primeras escuelas de Weißstadt en conseguir de modo

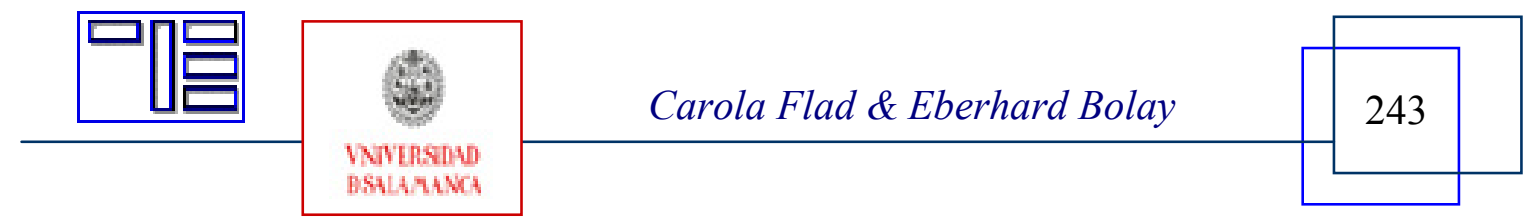


Revista Electrónica Teoría de la Educación.

Educación y Cultura en la Sociedad de la Información.

http://www.usal.es/teoriaeducacion

Vol. 9. No 2. Mayo 2008

activo el apoyo público para la juventud. Después de que la posición del trabajador social quedara vacante durante un año, fue ocupada en julio de 2001 por Tobias Herder. Su puesto está repartido en el 50\% de trabajo social en el contexto de la Hauptschule y el 50\% en el trabajo en la Casa de la Juventud vecina

5 Una argumentación similar se encuentra en Dohmen con respecto al apoyo del aprendizaje informal mediante instituciones educativas. "Se trata siempre de encontrar un equilibrio en situaciones de aprendizaje plausible que no anule la motivación entre un aprendizaje prospectivo, fundamental y más sistemát co, y un aprendizaje ad-hoc enfocado en intereses”. Domen, 2001 Op.cit. 136

6 Streblow (2003) describe tales procesos informales de aprendizaje como "aprendizaje salvaje“.

${ }^{7}$ Röll enfatiza en el papel de los maestros como administradores "omnicompetentes" de conocimiento (Röll 2002:18), que imposibilitan "vivencias de autonomía” (Ibid.:21) por parte de los alumnos y mantienen, en cambio, una "competencia incuestionada de evaluación" de los alumnos. La competencia de vida que ha sido adquirida por los alumnos más allá de condiciones pedagógicas firmes y que incorporan en esa experiencia, no sería recibida de manera constructiva, sino causaría malentendidos.

Este texto ya ha sido publicado como Flad, C./Bolay, E. 2006: Schulsozialarbeit aus der Perspektive von Schülerinnen und Schülern; en: Bitzan, M./Bolay, E./Thiersch, H. (edit.): Die Stimme der Adressaten. Empirische Forschung über Erfahrungen von Mädchen und Jungen mit der Jugendhilfe, Weinheim/München, p. 159-174

Para citar este artículo puede utilizar la siguiente referencia:

FLAD, Carola \& BOLAY, Eberhard (2008). Trabajo Social Escolar desde la perspectiva de alumnas y alumnos en Alemania. Un ejemplo de BadenWürtemberg. En APARICIO, Pablo (Coord.) Desde la diversidad hacia la desigualdad: ¿destino inexorable de la globalización? [monográfico en línea]. Revista Electrónica Teoría de la Educación: Educación y Cultura en la Sociedad de la Información. Vol. 8, $\mathrm{n}^{\circ}$ 2. Universidad de Salamanca. [Fecha de consulta: $\mathrm{dd} / \mathrm{mm} /$ aaaa $]$.

$<\mathrm{http} / /$ www.usal.es/ teoriaeducacion/rev_numero_09_02/n9_02_fladbolay.pdf > ISSN 1138-9737

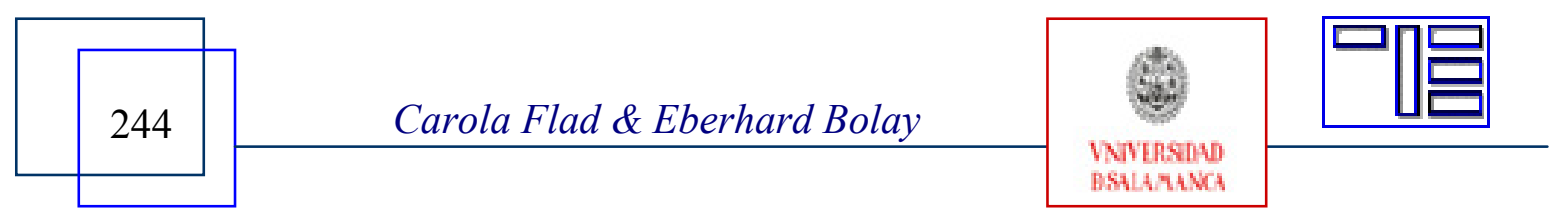

